\title{
Agradecimento aos Revisores da Revista Brasileira de Medicina de Família e Comunidade (RBMFC)
}

\section{Gustavo Diniz Ferreira Gusso, Thiago Dias Sarti}

A Revista Brasileira de Medicina de Família e Comunidade (RBMFC) agradece aos Avaliadores listados abaixo que atuaram como revisores ad hoc durante o ano de 2017, dedicando horas voluntariamente para a emissão de pareceres técnicos sobre manuscritos submetidos a esta revista.

1. Adhemar Dias Figueiredo/Hospital Municipal de Governador Valadares

2. Airton Tetelbom Stein/Universidade Federal de Ciências da Saúde de Porto Alegre (UFCSPA)

3. Alessandro da Silva Scholze/Universidade do Vale do Itajaí (UNIVALI)

4. Alessandro Leite Cavalcanti/Universidade Estadual da Paraíba (UEPB)

5. Aline Guerra Aquilante/Universidade Federal de São Carlos (UFSCar)

6. Amaury Lelis Dal-Fabbro/Universidade de São Paulo (USP

7. Ana Lucia Soares Camargo/Universidade do Extremo Sul Catarinense (UNESC)

8. Andréa Silvia de Walter/Universidade Federal do Ceará (UFC)

9. Angela Helena Marin/Universidade do Vale do Rio dos Sinos (UNISINOS)

10. Antônio Augusto Dall'Agnol Modesto/Universidade de São Paulo (USP)

11. Bruno José Barcellos Fontanella/Universidade Federal de São Carlos (UFSCar)

12. Carlos André Aita Schmitz/Universidade Federal do Rio Grande do Sul (UFRGS)

13. Carlos Eduardo Aguilera Campos/Universidade Federal do Rio de Janeiro (UFRJ)

14. Cesar Augusto Orazem Favoreto/Universidade do Estado do Rio de Janeiro (UERJ)

15. Charles Dalcanale Tesser/Universidade Federal de Santa Catarina (UFSC)

16. Daniel Almeida Gonçalves/Universidade Federal de São Paulo (UNIFESP)

17. Daniel Knupp Augusto/UNIMED, Belo Horizonte-MG

18. Deise Fernanda Peixoto Oliveira/UNIMED Sergipe

Como citar: Gusso GDF, Sarti TD. Agradecimento aos Revisores da Revista Brasileira de Medicina de Família e Comunidade (RBMFC). Rev Bras Med Fam Comunidade. 2017;12(39):1-3. http://dx.doi.org/10.5712/rbmfc12(39)1708 
19. Deoclecio Avigo/Universidade de São Paulo (USP)

20. Diego José Brandão/Universidade de Vila Velha (UVV)

21. Donovan Casas Patiño/Universidad Autnoma del Estado de Mexico

22. Eduardo Bertol/Secretaria Municipal de Saúde de Curitiba, PR

23. Elson Romeu Farias/Universidade Luterana do Brasil (ULBRA)

24. Fabio Vieira/Faculdade Evangélica de Medicina do Paraná (FEPAR)

25. Francisco Rogerlândio de Martins/Universidade Federal do Ceará (UFC)

26. Gabriel Hahn Monteiro Lufchitz/Clinica Nova Oncologia, Florianópolis, SC

27. Gabriela dos Santos Buccini/Universidade de São Paulo (USP)

28. Giannina do Espírito-Santo/Centro Universitário Augusto Motta (UNISUAM)

29. Hamilton Lima Wagner/Prefeitura Municipal de Curitiba, PR

30. Hernâni Pombas Caniço/Assistente Convidado da Faculdade de Medicina da Universidade de Coimbra

31. Isanete Geraldini Costa Bieski/Universidade Federal de Mato Grosso (UFMT)

32. János Valery Gyuricza/Fundação Faculdade de Medicina (FFM). UBS Jardim Boa Vista

33. João Werner Falk/Universidade Federal do Rio Grande do Sul (UFRGS)

34. Josenaide Engracia dos Santos/Universidade de Brasília (UNB), Campus Ceilândia. Brasília, DF

35. Juan Gérvas/Equipo CESCA, Madrid

36. Juliany Luz/Prefeitura Municipal de Biguaçu, Biguaçu, SC

37. Leonardo Cançado Monteiro Savassi/Universidade Federal de Ouro Preto - UFOP e Universidade Federal de Minas Gerais - UFMG

38. Leonardo Ferreira Fontenelle/Universidade Vila Velha

39. Leonardo Graever/Secretaria Municipal de Saúde do Rio de Janeiro (SMS-RJ)

40. Leonardo Silva Borges Roever/Universidade Federal de Uberlândia (UFU)

41. Leonardo Vieira Targa/Universidade de Caxias do Sul (UCS)

42. Lourdes Luzón Oliver/PRMFC do Municipio do Rio de Janeiro

43. Luciane Loures dos Santos/Universidade de São Paulo (FMRP-USP). Ribeirão Preto, SP

44. Luis Pisco/Instituto de Ciências Socias e Politicas (ISCSP)

45. Luiz Miguel Santiago/Universidade da Beira Interior (UBI)

46. Magda Moura Almeida Porto/Universidade de Fortaleza (UNIFOR)

47. Marcello Dala Bernardina Dalla/Universidade Vila Velha (UVV) 
48. Marcos Adams Goldraich/Secretaria Municipal de Saúde e Defesa Civil - Rio de Janeiro, SMSDC-RJ,

49. Margarita Silva Diercks/Grupo Hospitalar Conceição (GHC)

50. Maria Eugênia Bresolin Pinto/Universidade Federal de Ciências da Saúde de Porto Alegre (UFCSPA)

51. Maria Inez Padula Anderson/Universidade Estadual do Rio de Janeiro (UERJ

52. Maria Luciene Feitosa Rocha/Universidade Federal do Piauí. Floriano, PI

53. Mario Maia Bracco/Instituto Israelita de Ensino e Pesquisa Albert Einstein (IIEPAE). São Paulo, SP

54. Maristela Carbol/Universidade Federal de São Carlos (UFSCar)

55. Melanie Noël Maia/Secretaria Municipal de Saúde do Rio de Janeiro (SMS-RJ)

56. Mellina Marques Vieira Izecksohn/Centro de Saúde Escola Germano Sinval Faria (CSEGSF). Rio de Janeiro, RJ, Brasil

57. Nelson Robson Mendes/Universidade Estadual do Rio de Janeiro (UERJ)

58. Nilson Massakazu Ando/Secretaria Municipal de Saúde de Manaus (SEMSA)

59. Patricia Sampaio Chueiri/Ministério da Saúde (MS), Departamento de Atenção Básica (DAB)

60. Rafael Nicolau de Carvalho/Universidade Federal da Paraíba (UFPB)

61. Roberto Henrique Amorim/Universidade Federal do Rio Grande do Sul (UFRGS)

62. Rodolfo de Carvalho Pacagnella/Universidade Estadual de Campinas (UNICAMP). Campinas, SP

63. Rogério Dias Renovato/Universidade Estadual de Mato Grosso do Sul (UFMS)

64. Rogerio Luz Coelho/Residência em MFC - UFPR/SMS Curitiba/Faculdade Evangélica de Medicina do Paraná (FEPAR)

65. Rosana Pimentel Correia Moysés/Universidade Federal do Amazonas (UFAM)

66. Rosangela Ziggiotti Oliveira/Universidade Estadual de Maringá (UEM)

67. Ruth Borges Dias/Universidade José do Rosário Vellano (UNIFENAS)

68. Sandra Maria Spedo/Universidade Federal de São Paulo (UNIFESP)

69. Yuji Magalhães Ikuta/Universidade do Estado do Pará (UEPA) 